\title{
Análise dialélica parcial em gerações avançadas para seleção de populações segregantes de trigo
}

\author{
Adérico Júnior Badaró Pimentel(1), Moacil Alves de Souza(1), Pedro Crescêncio Souza Carneiro(1), \\ João Romero do Amaral Santos de Carvalho Rocha(1), Juarez Campolina Machado(2) e Guilherme Ribeiro ${ }^{(3)}$
}

(1)Universidade Federal de Viçosa, Campus Viçosa, Avenida P.H. Rolfs, s/no, CEP 36570-000 Viçosa, MG, Brasil. E-mail: adericojr@yahoo.com.br, moacil@ufv.br, carneiro@ufv.br, joao.rocha@ufv.br (2)Embrapa Gado de Leite, Rua Eugênio do Nascimento, no 610, Bairro Dom Bosco, CEP 36038-330 Juiz de Fora, MG, Brasil. E-mail: juarez.machado@embrapa.br (3)Universidade Federal do Pampa, Campus Itaqui, Rua Luiz Joaquim de Sá Britto, s/no, CEP 97650-000 Itaqui, RS, Brasil. E-mail: guilhermeribeiro@unipampa.edu.br

Resumo - O objetivo deste trabalho foi estimar, em gerações avançadas, os efeitos da capacidade geral e específica de combinação de genitores de trigo, bem como selecionar populações segregantes superiores. Doze genitores e suas 36 populações nas gerações $\mathrm{F}_{2}$ e $\mathrm{F}_{3}$, obtidas em arranjo de dialelo parcial, foram avaliados quanto à produtividade de grãos. Utilizou-se o delineamento em látice $7 \times 7$, com duas repetições, mais um tratamento para completar o látice. O efeito de capacidade geral de combinação foi significativo, nas duas gerações. Observou-se alta correlação $(0,83)$ dos efeitos de capacidade geral de combinação entre gerações, mas inexpressiva influência dos efeitos de gerações e de anos na classificação dos cruzamentos. As populações provenientes do cruzamento entre os genitores BRS 254, BRS 264 e IAC 364 (Tucuruí III) e os genitores MGS 1 Aliança, VI 98053 e UFVT 1 Pioneiro apresentam maior potencial para obtenção de linhagens superiores, quanto à produtividade de grãos. $\mathrm{O}$ uso da análise dialélica parcial em gerações avançadas é promissor para programas de melhoramento de trigo por hibridação.

Termos para indexação: Triticum aestivum, capacidade de combinação, melhoramento de plantas, predição do potencial genético.

\section{Partial diallel analysis in advanced generations for selection of wheat segregating populations}

\begin{abstract}
The objective of this work was to estimate the effects of general and specific combining ability of wheat parental lines in advanced generations, and to select superior segregating populations. Twelve parental lines and their 36 populations in $\mathrm{F}_{2}$ and $\mathrm{F}_{3}$ generations, obtained in a partial diallel arrangement, were evaluated for grain yield. The experimental design used was a $7 \times 7$ simple lattice with two replicates, plus a treatment to complete the lattice. The effect of general combining ability was significant in both generations. There was high correlation (0.83) of the effects of general combining ability between generations, but inexpressive influence of the effects of generations and years were observed on the classification of crossings. The populations derived from the crossings between the parental lines BRS 254, BRS 264, and IAC 364 (Tucuruí III) and the parental lines MGS 1 Aliança, VI 98053, and UFVT 1 Pioneiro show the greatest potential for obtaining superior lines for grain yield. The use of the partial diallel analysis in advanced generations is promising for wheat breeding programs through hybridization.
\end{abstract}

Index terms: Triticum aestivum, combining ability, crop breeding, prediction of genetic potential.

\section{Introdução}

No Brasil, o consumo de trigo (Triticum aestivum L.) é de aproximadamente $55 \mathrm{~kg}$ por habitante por ano, o que corresponde a um consumo interno anual em torno de 10,5 milhões de toneladas (CONAB, 2012). Porém, a produção nacional de trigo não supre a demanda interna, e cerca de $50 \%$ do trigo consumido no país é importado. Portanto, é premente a realização de pesquisas e incentivos à triticultura brasileira.
Pelo menos $50 \%$ do aumento de produtividade das principais espécies autógamas cultivadas no país, nos últimos 30 anos, é atribuído ao melhoramento genético (Ramalho \& Araújo, 2011). O sucesso dos programas de melhoramento está relacionado ao potencial das populações segregantes geradas nos cruzamentos (Kurek et al., 2001), determinado pela capacidade de combinação entre os genitores. Assim, a identificação de cruzamentos superiores constitui etapa crucial no processo de melhoramento (Kotzamanidis et al., 2008). 
A análise dialélica permite a seleção da melhor combinação de parentais, por meio da avaliação do comportamento das progênies oriundas dos cruzamentos. Além disso, ela permite determinar a ação gênica que controla o caráter (Cruz et al., 2012), o que auxilia na escolha do método de condução das populações e no critério de seleção a ser adotado.

Antes limitado ao melhoramento de espécies alógamas, esse método vem sendo utilizado em espécies autógamas, como arroz (Torres \& Geraldi, 2007; Pereira et al., 2008), feijão (Gonçalves-Vidigal et al., 2008; Trindade et al., 2011), aveia (Ribeiro et al., 2011) e trigo (Benin et al., 2009; Valério et al., 2009). Apesar do uso da capacidade de combinação como critério para seleção de cruzamentos em trigo, há pouca informação sobre o uso e a repetibilidade de suas estimativas obtidas em gerações com diferentes graus de endogamia.

Na cultura do trigo, a utilização da análise dialélica é limitada pela pequena quantidade de sementes disponível na geração $F_{1}$, em razão da dificuldade na realização dos cruzamentos e do número limitado de sementes híbridas obtidas por polinização. Uma alternativa é a avaliação do dialelo em gerações avançadas. De acordo com Bhullar et al. (1979), as gerações $F_{2}$ e $F_{3}$ podem ser utilizadas com eficiência para estudar a capacidade de combinação em trigo, pois resultam em melhores predições do que as obtidas com a geração $F_{1}$. Javaid et al. (2001) também destacam a eficiência da geração $F_{2}$ na avaliação da capacidade de combinação em trigo.

Estudos da capacidade de combinação conduzidos nas gerações $F_{2}$ ou $F_{3}$ oferecem, ainda, a oportunidade de testar o material em diferentes ambientes, o que possibilita a obtenção de informações sobre uma possível interação genótipo x ambientes (Bhullar et al., 1979; Masood \& Kronstad, 2000). A importância dessa análise é evidenciada no trabalho de Gowda et al. (2012), que evidencia que o valor e a significância do componente de variância da capacidade geral e específica de combinação são dependentes do local de avaliação. Dessa forma, unidades experimentais mais representativas e maior número de repetições podem ser utilizados nos delineamentos experimentais com gerações avançadas, uma vez que a disponibilidade de sementes é maior.

Como em gerações avançadas o desvio de dominância é reduzido, há possibilidade de o efeito da capacidade específica de combinação não ser significativo. Isso inviabilizaria a utilização de um dialelo completo, pois a informação sobre a complementaridade gênica entre os genitores seria perdida. Nessas condições, o uso do dialelo parcial poderia ser mais adequado. Esse dialelo consiste no cruzamento entre dois grupos de genitores complementares para características de interesse; desta forma, não existe interesse no cruzamento entre indivíduos do mesmo grupo. Segundo Viana (2007), nos dialelos parciais, a magnitude da capacidade geral de combinação, além de mostrar a frequência de alelos favoráveis, é indicativa da diversidade genética entre o genitor de um grupo e aqueles do grupo oposto.

O objetivo deste trabalho foi estimar, em gerações avançadas, os efeitos da capacidade geral e específica de combinação de genitores de trigo, por meio da análise dialélica parcial, bem como selecionar populações segregantes superiores.

\section{Material e Métodos}

Os experimentos foram realizados na Estação Experimental Prof. Diogo Alves de Mello, pertencente à Universidade Federal de Viçosa, MG $\left(20^{\circ} 45^{\prime} 14^{\prime \prime} \mathrm{S}\right.$, $42^{\circ} 52^{\prime} 55^{\prime \prime} \mathrm{W}$, a $648 \mathrm{~m}$ de altitude).

Para a obtenção das sementes $F_{1}, 12$ linhagens elites foram cruzadas em arranjo de dialelo parcial. Os genitores foram estratificados em dois grupos (Tabela 1). O grupo 1 foi constituído por linhagens classificadas como Trigo Melhorador, exceto a cultivar BRS 264; o grupo 2 foi constituído por linhagens tolerantes ao calor (Anahuac 75 e MGS 1 Aliança), de alta estabilidade de produção (VI 98053 e UFVT 1 Pioneiro) e de alto potencial produtivo e boa estatura (BRS 207 e IVI 01041).

As sementes $F_{1}$ foram multiplicadas em casa de vegetação, em 2007, e, no ano seguinte, foram avaliados os 12 genitores e suas 36 populações $F_{2}$. Utilizou-se o delineamento em látice $7 \times 7$, com duas repetições, mais um tratamento para completar o látice. As parcelas foram constituídas por duas linhas de 1,0 m de comprimento espaçadas em $0,20 \mathrm{~m}$, com densidade de 300 sementes aptas por metro quadrado.

Em 2009, foram avaliados os 12 genitores e as 36 populações $\mathrm{F}_{3}$, em que as sementes utilizadas foram obtidas por amostragem da geração anterior colhida em bulk. O delineamento experimental, o espaçamento e a densidade de semeadura foram os mesmos empregados 
na geração anterior. A parcela foi constituída por cinco linhas de 5,0 $\mathrm{m}$ de comprimento, e as três linhas centrais constituíram a área útil.

A semeadura foi realizada na segunda quinzena de maio, para os dois experimentos. Utilizou-se $250 \mathrm{~kg} \mathrm{ha}^{-1}$ da fórmula N-P-K (08-28-16), no sulco de semeadura, e $250 \mathrm{~kg} \mathrm{ha}^{-1}$ de sulfato de amônio, aplicado em cobertura no início do perfilhamento. Para o controle de plantas invasoras, foi realizada a aplicação de $5 \mathrm{~g} \mathrm{ha}^{-1}$ de produto comercial do herbicida Ally (metsulfuron metílico), aos 15 dias após a semeadura. $\mathrm{O}$ cultivo foi conduzido sob irrigação por aspersão convencional.

Avaliou-se a produtividade de grãos, tendose determinado a produção total de cada parcela transformada para $\mathrm{kg} \mathrm{ha} \mathrm{h}^{-1}$. A pesagem foi realizada após o beneficiamento e a secagem dos grãos até aproximadamente $13 \%$ de umidade. Os dados foram submetidos à análise de variância segundo o modelo em látice. A decisão de analisar os experimentos delineados em látice como intrablocos do látice, com tratamentos ajustados, ou como blocos completos ao acaso foi feita conforme as recomendações de Federer (1955) e Silva et al. (2000). De acordo com Silva et al. (2000), o modelo de análise intrablocos de experimentos em látice, para estimar componentes da variância, deve ser utilizado quando a eficiência relativa do delineamento em látice for superior a $100 \%$, em comparação ao delineamento em blocos completos ao acaso. Caso contrário, deve-se optar pelo modelo de análise em blocos. Federer (1955) recomenda que a análise dos dados seja realizada em látice somente se a eficiência for superior a $110 \%$.

A análise dialélica para cada geração foi realizada segundo o modelo de Geraldi \& Miranda Filho (1988), para dialelo parcial, adaptado do método de Griffing (1956):

$$
Y_{i j}=\mu+1 / 2\left(d_{1}+d_{2}\right)+g_{i}+g_{j}^{\prime}+s_{i j}+\varepsilon_{i j},
$$

em que $Y_{\mathrm{ij}}$ é a média do cruzamento que inclui o i-ésimo genitor do grupo 1 e o j-ésimo genitor do grupo $2 ; \mu$ é a média geral do dialelo; $d_{1}$ e $d_{2}$ são os contrastes que incluem as médias dos grupos 1 e 2 e a média geral; $g_{\mathrm{i}}$ é o efeito da capacidade geral de combinação do i-ésimo genitor do grupo $1 ; \mathrm{g}_{\mathrm{j}}^{\prime}$ é o efeito da capacidade geral de combinação do j-ésimo genitor do grupo $2 ; \mathrm{s}_{\mathrm{ij}}$ é o efeito da capacidade específica de combinação; e $\varepsilon_{\mathrm{ij}}$ é o erro experimental médio.

O desvio-padrão das estimativas de capacidade geral $\left(\hat{\mathrm{g}}_{\mathrm{i}}\right.$ e $\left.\hat{\mathrm{g}}_{\mathrm{j}}\right)$ e específica de combinação $\left(\hat{\mathrm{s}}_{\mathrm{ij}}\right)$ foi obtido pela raiz quadrada das variâncias, conforme Cruz et al. (2012). As análises estatísticas foram realizadas com o auxílio do programa Genes (Cruz, 2006).

\section{Resultados e Discussão}

A eficiência do delineamento em látice, em comparação ao de blocos casualizados, foi verificada apenas na geração $\mathrm{F}_{2}$, e apresentou valor baixo (11\%). Portanto, optou-se pelas análises segundo o modelo de blocos ao acaso. Os coeficientes de variação foram de $17 \%$, no experimento com a geração $\mathrm{F}_{2}$, e de $13 \%$, com a geração $\mathrm{F}_{3}$, classificados como médios e

Tabela 1. Relação dos genitores de trigo, cruzados em arranjo de dialelo parcial, para obtenção das populações segregantes.

\begin{tabular}{|c|c|c|}
\hline Genitores & Cruzamento & Instituição \\
\hline \multicolumn{3}{|c|}{ Grupo 1} \\
\hline Embrapa 22 & Veery“S”/3/Klein Toledo“S”/PAT 19//Moncho/ Jupateco 73 & Embrapa \\
\hline Embrapa 42 & LAP 689/MS 7936 & Embrapa \\
\hline BRS 254 & Embrapa $22 * 3 /$ Anahuac 75 & Embrapa \\
\hline BRS 264 & Buckbuck/Chiroca//Tui & Embrapa \\
\hline IAC 24 (Tucuruí) & IAS 51/SON 64/YAQUI 50E/GB/2*CIANO & IAC \\
\hline IAC 364 (Tucuruí III) & IAS 58/IAS 55//IALS“S”/3/IAC 5/4/ALD“S”//IAS 58/8.1034.A//ALD/5/CNR/6/BUC/7/IAC 24 & IAC \\
\hline \multicolumn{3}{|c|}{ Grupo 2} \\
\hline Anahuac 75 & II 12300//Lerma Rojo 64/8156/3/Norteno 67 & CIMMYT \\
\hline MGS 1 Aliança & PF 858/OCEPAR 11 & Epamig/UFV \\
\hline BRS 207 & Seri 82/PF 813 & Embrapa \\
\hline VI 98053 & Embrapa 22/BR 12 & UFV \\
\hline UFVT 1 Pioneiro & Veery $5 /$ NACOZARI & UFV \\
\hline IVI 01041 & TRAP\#1/YACO/3/KAUZ*2/TRAP//KAUZ & UFV \\
\hline
\end{tabular}


rotineiramente observados em ensaios em campo com trigo (Lúcio et al., 1999).

A análise dialélica detectou efeito significativo dos tratamentos, em ambas as gerações (Tabela 2). Assim, a soma de quadrados de tratamentos foi desdobrada em soma de quadrados de grupos (G1 x G2), soma de quadrados da capacidade geral de combinação do grupo 1 (CGC 1) e do grupo 2 (CGC 2), e soma de quadrados da capacidade específica de combinação (CEC).

Não houve efeito significativo dos grupos, o que evidencia que o número médio de alelos favoráveis é similar nos dois grupos de genitores (Tabela 2). Esse fato decorre da presença, em ambos os grupos, de cultivares produtivas, recomendadas para cultivo, e de linhagens elite, em fase final de avaliação.

Os quadrados médios referentes à CGC dos grupos 1 e 2 foram significativos, nas duas gerações, o que mostra a existência de número diferente de alelos favoráveis entre os genitores dentro de cada grupo (Tabela 2). No entanto, em nenhuma das gerações houve efeito significativo da CEC, o que indica ausência de variabilidade para essa fonte de variação.

A não significância dos efeitos da CEC pode ser explicada a partir da expressão utilizada para sua obtenção. O efeito da CEC $\left(\hat{\mathrm{s}}_{\mathrm{ij}}\right)$ do i-ésimo com o j-ésimo genitor, relativamente a um loco, é dado por $\hat{\mathrm{S}}_{\mathrm{ij}}=2\left[\left(\overline{\mathrm{p}}-\mathrm{p}_{\mathrm{i}}\right)\left(\mathrm{t}_{\mathrm{j}}-\overline{\mathrm{t}}\right)\right] \mathrm{d}$, em que $\overline{\mathrm{p}}$ é a frequência média de alelos do grupo $1 ; \mathrm{p}_{\mathrm{i}}$ é a frequência do alelo favorável no i-ésimo pai do grupo $1 ; t_{j}$ é a frequência do alelo favorável no j-ésimo pai do grupo $2 ; \overline{\mathrm{t}}$ é a frequência média de alelos do grupo 2; e d é o efeito dos locos em heterozigose. Portanto, a CEC é função dos desvios de

Tabela 2. Resumo das análises dialélicas para produtividade de grãos de populações segregantes de trigo, nas gerações $F_{2}$ e $\mathrm{F}_{3}$ e em seus genitores.

\begin{tabular}{lccc}
\hline Fonte de variação $^{(1)}$ & Grau de & \multicolumn{2}{c}{ Quadrado médio } \\
\cline { 3 - 4 } & liberdade & $\mathrm{F}_{2}$ & $\mathrm{~F}_{3}$ \\
\hline Tratamentos & 47 & $1.655 .583,35^{* *}$ & $330.912,44^{* *}$ \\
Grupos (G1 x G2) & 1 & $21.122,67^{\mathrm{ns}}$ & $3.151,50^{\mathrm{ns}}$ \\
CGC 1 & 5 & $1.720 .971,79^{*}$ & $659.213,33^{* *}$ \\
CGC 2 & 5 & $9.408 .694,67^{* *}$ & $1.152 .367,16^{* *}$ \\
CEC & 36 & $615.082,30^{\mathrm{ns}}$ & $180.328,63^{\mathrm{ns}}$ \\
Resíduo & 47 & $594.663,50$ & $110.024,83$ \\
\hline
\end{tabular}

${ }^{(1)}$ CGC 1, capacidade geral de combinação do grupo 1; CGC 2, capacidade geral de combinação do grupo 2; CEC, capacidade específica de combinação. ** e *Significativo a 1 e $5 \%$ de probabilidade, respectivamente, pelo teste F. ns Não significativo. dominância e da diversidade genética entre os genitores (Vencovsky, 1978). Como os pais são divergentes para o caráter em questão (Pimentel et al., 2013), a não significância dos efeitos da CEC (Tabela 3) pode ser resultante da pequena contribuição dos efeitos de dominância para expressão do caráter nas gerações analisadas, nas quais a heterose é reduzida a $1 / 2\left(\mathrm{~F}_{2}\right)$ e $1 / 4\left(F_{3}\right)$ do seu valor inicial. Contudo, a explicação com base na ausência de dominância é pouco provável, uma

Tabela 3. Efeito da capacidade específica de combinação $\left(\hat{s}_{\mathrm{ij}}\right)$ quanto à produtividade de grãos, na avaliação de genitores e populações segregantes de trigo, nas gerações $F_{2}$ e $F_{3}$.

\begin{tabular}{|c|c|c|}
\hline Cruzamento & Geração $F_{2}$ & Geração $F_{3}$ \\
\hline Embrapa $22 \times$ Anahuac 75 & 100,15 & 586,02 \\
\hline Embrapa 22 x MGS 1 Aliança & 68,45 & $-265,41$ \\
\hline Embrapa 22 x BRS 207 & $-224,24$ & $-529,56$ \\
\hline Embrapa 22 x VI 98053 & $-47,74$ & $-78,21$ \\
\hline Embrapa 22 x IVI 01041 & 514,15 & 264,69 \\
\hline Embrapa 22 x UFVT 1 Pioneiro & 631,25 & 323,29 \\
\hline Embrapa 42 x Anahuac 75 & $-838,34$ & 158,10 \\
\hline Embrapa 42 x MGS 1 Aliança & 256,96 & 308,33 \\
\hline Embrapa 42 x BRS 207 & $-252,74$ & $-56,66$ \\
\hline Embrapa 42 x VI 98053 & $-386,24$ & $-389,48$ \\
\hline Embrapa 42 x IVI 01041 & 36,65 & $-459,91$ \\
\hline Embrapa 42 x UFVT 1 Pioneiro & 493,75 & $-206,14$ \\
\hline BRS 254 x Anahuac 75 & $-598,14$ & $-179,33$ \\
\hline BRS 254 x MGS 1 Aliança & 148,16 & $-292,93$ \\
\hline BRS 254 x BRS 207 & $-373,54$ & 245,26 \\
\hline BRS 254 x VI 98053 & $-5,04$ & 349,94 \\
\hline BRS 254 x IVI 01041 & 820,85 & $-180,82$ \\
\hline BRS 254 x UFVT 1 Pioneiro & $-581,04$ & $-227,56$ \\
\hline BRS 264 x Anahuac 75 & 610,25 & 17,13 \\
\hline BRS 264 x MGS 1 Aliança & 225,55 & $-53,81$ \\
\hline BRS 264 x BRS 207 & $-245,14$ & $-12,45$ \\
\hline BRS 264 x VI 98053 & 662,35 & 536,40 \\
\hline BRS 264 x IVI 01041 & $-837,74$ & $-356,54$ \\
\hline BRS 264 x UFVT 1 Pioneiro & $-609,64$ & $-19,61$ \\
\hline IAC 24 (Tucuruí) x Anahuac 75 & 786,95 & $-94,48$ \\
\hline IAC 24 (Tucuruí) x MGS 1 Aliança & 455,25 & 267,59 \\
\hline IAC 24 (Tucuruí) x BRS 207 & $1.010,55$ & 79,94 \\
\hline IAC 24 (Tucuruí) x VI 98053 & $-367,94$ & $-92,71$ \\
\hline IAC 24 (Tucuruí) x IVI 01041 & $-216,04$ & $-131,98$ \\
\hline IAC 24 (Tucuruí) x UFVT 1 Pioneiro & $-683,94$ & $-169,88$ \\
\hline IAC 364 (Tucuruí III) x Anahuac 75 & $-439,64$ & 66,29 \\
\hline IAC 364 (Tucuruí III) x MGS 1 Aliança & $-516,34$ & $-103,97$ \\
\hline IAC 364 (Tucuruí III) x BRS 207 & 629,95 & 105,20 \\
\hline IAC 364 (Tucuruí III) x VI 98053 & $-248,54$ & $-202,93$ \\
\hline IAC 364 (Tucuruí III) x IVI 01041 & $-544,64$ & $-372,37$ \\
\hline IAC 364 (Tucuruí III) x UFVT 1 Pioneiro & $-307,54$ & 150,56 \\
\hline Desvio-padrão $\left(\hat{s}_{\mathrm{ij}}\right)$ & 491,51 & 211,42 \\
\hline
\end{tabular}


vez que há heterose para produtividade de grãos em trigo (Farooq \& Khaliq, 2004; Bertan et al., 2009).

Os resultados obtidos nas duas gerações $\left(\mathrm{F}_{2}\right.$ e $\mathrm{F}_{3}$ ) foram análogos. Além da ausência de efeitos significativos da CEC, observou-se estimativa do coeficiente de correlação de Spearman de 0,83 , entre os efeitos de CGC estimados nas gerações $\mathrm{F}_{2}$ e $\mathrm{F}_{3}$ (Tabela 4). Esses resultados indicam inexpressiva influência dos efeitos de gerações e de anos nas estimativas dos parâmetros genéticos, e são evidência de que a seleção com base na geração $F_{2}$ ou $F_{3}$ conduz a resultados semelhantes. Portanto, a análise dialélica parcial em gerações avançadas, em especial na $\mathrm{F}_{3}$, cuja disponibilidade de sementes é maior, é promissora na escolha de potenciais genitores de trigo.

Resultados semelhantes foram obtidos por Masood \& Kronstad (2000). Os autores compararam a análise dialélica nas gerações $F_{1}, F_{2}$ e $\quad F_{3}$ e concluíram que a capacidade geral de combinação foi amplamente consistente ao longo das gerações, para o caráter produção de grãos e seus componentes em trigo. As estimativas da capacidade específica de combinação mostraram-se pouco consistentes, e de baixa repetibilidade, ao longo das gerações. Segundo Bornhofen et al. (2013), selecionar cruzamentos de trigo que apresentem genitores com capacidade geral de combinação superior maximiza o rendimento de

Tabela 4. Efeito da capacidade geral de combinação dos genitores dos grupos $1\left(\hat{\mathrm{g}}_{\mathrm{i}}\right)$ e $2\left(\hat{\mathrm{g}}_{\mathrm{j}}\right)$, para produtividade de grãos, obtidos na avaliação de genitores e populações segregantes de trigo nas gerações $\mathrm{F}_{2}$ e $\mathrm{F}_{3}$.

\begin{tabular}{lrr}
\hline Genitores & Geração $\mathrm{F}_{2}$ & Geração $\mathrm{F}_{3}$ \\
\hline \multicolumn{3}{c}{ Grupo 1 } \\
Embrapa 22 & $-7,62$ & $-97,57$ \\
Embrapa 42 & $-282,12$ & $-244,80$ \\
BRS 254 & 106,68 & 54,28 \\
BRS 264 & 447,28 & 237,16 \\
IAC 24 (Tucuruí) & $-360,42$ & $-106,57$ \\
IAC 364 (Tucuruí III) & 96,18 & 157,50 \\
\hline & & Grupo 2 \\
Anahuac 75 & $-410,47$ & $-230,72$ \\
MGS 1 Aliança & 903,22 & 288,05 \\
BRS 207 & $-852,07$ & $-314,64$ \\
VI 98053 & 682,42 & 115,01 \\
IVI 01041 & $-410,48$ & $-50,39$ \\
UFVT 1 Pioneiro & 87,42 & 192,68 \\
\hline Desvio-padrão $\left(\hat{g}_{i}\right.$ e $\left.\hat{g}_{j}\right)$ & 157,41 & 67,71
\end{tabular}

grãos por planta, o número de grãos por espiga e a estatura de planta, independentemente da geração avaliada. De acordo com Bhullar et al. (1979), no entanto, quando o objetivo for avaliar o desempenho de híbridos de trigo, o material deve ser estudado na geração $F_{1}$. Contudo, para predizer o desempenho de populações em $F_{5}$, as gerações $F_{2}$ ou $F_{3}$ são as que propiciam estimativas mais confiáveis.

A análise dialélica em gerações avançadas é justificada em razão da possibilidade de ocorrer viés nas estimativas de CGC, quando se utiliza a geração $F_{1}$. No dialelo parcial, a CGC é função dos desvios de dominância, conforme evidenciado pela expressão apresentada por Vencovsky (1978): $\mathrm{CGC}_{\mathrm{i}}=\left(\mathrm{p}_{\mathrm{i}}-\overline{\mathrm{p}}\right)[\mathrm{a}+(1-2 \overline{\mathrm{t}}) \mathrm{d}]$, em que d é o efeito dos locos em heterozigose. Assim, a existência de desvios de dominância expressivos pode causar viés nas estimativas da CGC e induzir a erros na seleção de genitores e cruzamentos superiores.

Efeitos gênicos aditivos e não aditivos, no controle da expressão da produção de grãos em trigo, foram verificados na geração $F_{1}$, por Kamaluddin et al. (2007), e nas gerações $F_{1}$ e $F_{2}$ por Joshi et al. (2004). Zeeshan et al. (2013) constataram, por meio de análise dialélica, contribuição de efeitos gênicos não aditivos na geração $\mathrm{F}_{1}$ superior a dos efeitos aditivos, para produção de grãos e seus componentes. Essas evidências justificam a utilização do dialelo parcial em gerações avançadas, conforme proposto no presente trabalho, uma vez que os desvios de dominância diminuem com o avanço das gerações.

Cabe ressaltar que a escolha dos potenciais genitores na análise dialélica parcial, em gerações avançadas, deve-se basear apenas nas estimativas de CGC. Isso porque, neste caso, a magnitude da CGC é indicativa tanto da frequência de alelos favoráveis quanto da diversidade genética entre os genitores de um grupo e aqueles do outro grupo.

Com base nas estimativas de CGC obtidas na geração $\mathrm{F}_{3}$, as populações oriundas dos cruzamentos das cultivares BRS 254, BRS 264 e IAC 364 (Tucuruí III), pertencentes ao grupo 1, com as cultivares MGS 1 Aliança, UFVT 1 Pioneiro e VI 98053, pertencentes ao grupo 2, apresentaram maior potencial para obtenção de linhagens superiores, pois, além de conterem maior número de alelos favoráveis, elas foram as mais divergentes (Tabela 4).

Pesq. agropec. bras., Brasília, v.48, n.12, p.1555-1561, dez. 2013 DOI: 10.1590/S0100-204X2013001200004 


\section{Conclusões}

1. O uso da análise dialélica parcial em gerações avançadas é promissor para programas de melhoramento de trigo por hibridação.

2. A capacidade de combinação entre genitores é análoga nas gerações $\mathrm{F}_{2}$ e $\mathrm{F}_{3}$.

3. Os efeitos gênicos aditivos são os responsáveis pela expressão do caráter produtividade de grãos, nas gerações $\mathrm{F}_{2}$ e $\mathrm{F}_{3}$.

4. As populações provenientes do cruzamento entre os genitores BRS 254, BRS 264 e IAC 364 (Tucuruí III) e os genitores MGS 1 Aliança, VI 98053 e UFVT 1 Pioneiro apresentam maior potencial para obtenção de linhagens superiores, para o caráter produtividade de grãos.

\section{Agradecimentos}

Ao Conselho Nacional de Desenvolvimento Científico e Tecnológico (CNPq) e à Coordenação de Aperfeiçoamento de Pessoal de Nível Superior (Capes), pelo apoio financeiro.

\section{Referências}

BENIN, G.; SILVA, G.O. da; PAGLIOSA, E.S.; LEMES, C.; SIGNORINI, A.; BECHE, E.; CAPELIN, M.A. Capacidade de combinação em genótipos de trigo estimada por meio de análise multivariada. Pesquisa Agropecuária Brasileira, v.44, p.1145-1151, 2009. DOI: 10.1590/S0100-204X2009000900012.

BERTAN, I.; CARVALHO, F.I.F. de; OLIVEIRA, A.C. de; SILVA, J.A.G. da; BENIN, G.; HARTWIG, I.; SCHMIDT, D.A.M.; VALÉRIO, I.P.; FONSECA, D.R. da; SILVEIRA, G. Efeitos da heterose e endogamia em caracteres de importância agronômica em trigo. Revista Ceres, v.56, p.753-763, 2009.

BHULLAR, G.S.; GILL, K.S.; KHEHRA, A.S. Combining ability analysis over F1-F5 generations in diallel crosses of bread wheat. Theoretical and Applied Genetics, v.55, p.77-80, 1979. DOI: 10.1007/BF00285194

BORNHOFEN, E.; BENIN, G.; MATEI, G.; SILVA, C.L. da; BECHE, E.; PAGLIOSA, E.S.; HAGEMANN, T.R.; PINNOW, C. Capacidade de combinação entre genitores de trigo em duas gerações. Semina: Ciências Agrárias, v.34, p.3129-3140, 2013. DOI: 10.5433/1679-0359.2013v34n6Supl1p3129.

COMPANHIA NACIONALDEABASTECIMENTO. Indicadores da agropecuária. Brasília: CONAB, 2012. Disponível em: $<$ http:// www.conab.gov.br>. Acesso em: 12 jun. 2012.

CRUZ, C.D. Programa Genes: biometria. Viçosa: Ed. da UFV, 2006. 382p

CRUZ, C.D.; REGAZZI, A.J.; CARNEIRO, P.C.S. Modelos biométricos aplicados ao melhoramento genético. 4.ed. Viçosa: Ed. da UFV, 2012. 514p.
FAROOQ, J.; KHALIQ, I. Estimation of heterosis and heterobeltiosis of some quantitative characters in bread wheat crosses. Asian Journal of Plant Sciences, v.3, p.508-511, 2004. DOI: 10.3923/ajps.2004.508.511.

FEDERER, W.T. Experimental designs. New York: The MacMillan, 1955. 544p.

GERALDI, I.O.; MIRANDA-FILHO, J.B. Adapted models for the analysis of combining ability of varieties in partial diallel crosses. Revista Brasileira de Genética, v.11, p.419-430, 1988.

GONÇALVES-VIDIGAL, M.C.; SILVÉRIO, L.; ELIAS, H.T.; VIDIGAL FILHO, O.S.; KVITSCHAL, M.V.; RETUCI, V.S.; SILVA, C.R. da. Combining ability and heterosis in common bean cultivars. Pesquisa Agropecuária Brasileira, v.43, p.1143-1150, 2008. DOI: 10.1590/S0100-204X2008000900007.

GOWDA, M.; LONGIN, C.F.H.; LEIN, V.; REIF, J.C. Relevance of specific versus general combining ability in winter wheat. Crop Science, v.52, p.2494-2500, 2012. DOI: 10.2135/ cropsci2012.04.0245.

GRIFFING, B. Concept of general and specific combining ability in relation to diallel crossing systems. Australian Journal of Biological Sciences, v.9, p.463-493, 1956.

JAVAID, A.; MASOOD, S.; MINHAS, N.M. Analysis of combining ability in wheat (Triticum aestivum L.) using $\mathrm{F}_{2}$ generation. Pakistan Journal of Biological Sciences, v.4, p.1303-1305, 2001. DOI: 10.3923/pjbs.2001.1303.1305.

JOSHI, S.K.; SHARMA, S.N.; SINGHANIA, D.L.; SAIN, R.S. Combining ability in the $F_{1}$ and $F_{2}$ generations of diallel cross in hexaploid wheat (Triticum aestivum L. em. Thell). Hereditas, v.141, p.115-121, 2004. DOI: 10.1111/j.1601-5223.2004.0173 $0 . x$.

KAMALUDDIN, R.M.; SINGH, L.C.P.; MALIK, Z.; JOSHI, A.K. Combining ability analysis for grain filling duration and yield traits in spring wheat (Triticum aestivum L. em. Thell.). Genetics and Molecular Biology, v.30, p.411-416, 2007. DOI: 10.1590/ S1415-47572007000300018.

KOTZAMANIDIS, S.T.; LITHOURGIDIS, A.S.; MAVROMATIS, A.G.; CHASIOTI, D.I.; ROUPAKIAS, D.G. Prediction criteria of promising $\mathrm{F}_{3}$ populations in durum wheat: a comparative study. Field Crops Research, v.107, p.257-264, 2008. DOI: 10.1016/j. fcr.2008.02.012.

KUREK, A.J.; CARVALHO, F.I.F.; ASSMANN, I.C.; CRUZ, P.J. Capacidade combinatória como critério de eficiência na seleção de genitores em feijoeiro. Pesquisa Agropecuária Brasileira, v.36, p.645-651, 2001. DOI: 10.1590/S0100-204X2001000400007.

LÚCIO, A.D.; STORCK, L.; BANZATTO, D.A. Classificação dos experimentos de competição de cultivares quanto a sua precisão. Pesquisa Agropecuária Gaúcha, v.5, p.99-103, 1999.

MASOOD, M.S.; KRONSTAD, W.E. Combining ability analysis over various generations in a diallel cross of bread wheat. Pakistan Journal of Agricultural Research, v.16, p.1-4, 2000.

PEREIRA, J.A.; MORAIS, O.P.; BRESEGHELLO, F. Análise da heterose de cruzamentos entre variedades de arroz-vermelho. Pesquisa Agropecuária Brasileira, v.43, p.1135-1142, 2008. DOI: $10.1590 / \mathrm{S} 0100-204 X 2008000900006$. 
PIMENTEL, A.J.B.; RIBEIRO, G.; SOUZA, M.A. de; MOURA, L.M.; ASSIS, J.C. de; MACHADO, J.C. Comparação de métodos de seleção de genitores e populações segregantes aplicados ao melhoramento de trigo. Bragantia, v.72, p.113-121, 2013. DOI: 10.1590/S0006-87052013005000026.

RAMALHO, M.A.P.;ARAÚJO, L.C.A. de. Breeding self-pollinated plants. Crop Breeding and Applied Biotechnology, v.11, p.1-7, 2011. DOI: $10.1590 /$ S1984-70332011000500002.

RIBEIRO, G.; SILVEIRA, G. da; CRESTANI, M.; NORNBERG, R.; LUCHE, S.H.; MEZZALIRA, I.; CARVALHO, F.I.F.; OLIVEIRA, A.C. Diallel analysis in white oat cultivars subjected to water stress. Crop Breeding and Applied Biotechnology, v.11, p.125-132, 2011. DOI: 10.1590/S1984-70332011000200004.

SILVA, H.D.; FERREIRA, D.F.; PACHECO, C.A.P. Avaliação de quatro alternativas de análise de experimentos em látice quadrado, quanto à estimação de componentes de variância. Bragantia, v.59, p.117-123, 2000. DOI: 10.1590/S0006-87052000000100018.

TORRES, E.A.; GERALDI, I.O. Partial diallel analysis of agronomic characters in rice (Oryza sativa L.). Genetics and Molecular Biology, v.30, p.605-613, 2007. DOI: 10.1590/ S1415-47572007000400018.
TRINDADE, R. dos S.;AMARAL JÚNIOR,A.T. do; RODRIGUES, R.; VIANA, J.M.S.; PEREIRA, M.G.; GONÇALVES, L.S.A. Combining ability for morphoagronomic traits in common bean and snap bean. African Journal of Agricultural Research, v.29, p.6240-6245, 2011.

VALÉRIO, I.P.; CARVALHO, F.I.F. de; OLIVEIRA, A.C. de; SOUZA, V.Q.; BENIN, G.; SCHMIDT, D.A.M.; RIBEIRO, G.; NORNBERG, R.; LUCH, H. Combining ability of wheat genotypes in two models of diallel analysis. Crop Breeding and Applied Biotechnology, v.9, p.100-107, 2009. DOI: 10.12702/1984-7033. v09n02a01.

VENCOVSKY, R. Herança quantitativa. In: PATERNIANI, E. (Ed.). Melhoramento e produção do milho no Brasil. Campinas: Fundação Cargill, 1978. p.122-195.

VIANA, J.M.S. Heterosis and combining ability analyses from the partial diallel. Bragantia, v.66, p.641-647, 2007. DOI: 10.1590/ S0006-87052007000100007.

ZEESHAN, M.; ARSHAD, W.; ALI, S.; TARIQ, M.; HUSSAIN, M.; SIDDIQUE, M. Estimation of combining ability effects for some yield related metric traits in intra-specific crosses among different spring wheat (Triticum aestivum L.) genotypes. International Journal of Advanced Research, v.1, p.6-10, 2013.

Recebido em 9 de setembro de 2013 e aprovado em 29 de novembro de 2013

Pesq. agropec. bras., Brasília, v.48, n.12, p.1555-1561, dez. 2013 DOI: 10.1590/S0100-204X2013001200004 